\title{
RELACIÓN ENTRE LA PRESENCIA DE CAMBIOS ÓSEOS DE OSTEOARTROSIS DEL CÓNDILO MANDIBULAR E INCLINACIÓN DE LA EMINENCIA ARTICULAR EN PACIENTES CLASE II ESQUELÉTICA EVALUADOS MEDIANTE TOMOGRAFÍA COMPUTARIZADA DE HAZ CÓNICO
}

RELATIONSHIP BETWEEN THE PRESENCE OF OSTEOARTHRITIC BONE CHANGES OF THE MANDIBULAR CONDYLE AND INCLINATION OF THE ARTICULAR EMINENCE IN SKELETAL CLASS II PATIENTS EVALUATED BY CONE BEAM COMPUTED TOMOGRAPHY

\author{
Víctor Pardo Juárez ${ }^{1}$ \\ drvictorpardo@gmail.com \\ ORCID: 0000-0003-2836-2946
}

\section{RESUMEN}

Objetivo: Identificar la relación entre la inclinación de la eminencia articular de pacientes clase II esquelética con signos de osteoartrosis, medidos con tomografía computarizada de haz cónico (TCHC) en pacientes del Centro de Diagnóstico por Imágenes (CDI) en Lima, Perú, entre los años 2010 y 2014. Materiales y método: El grupo de estudio retrospectivo será planificado de acuerdo con el tamaño de muestra de estimación y consistirá en exploraciones con TCHC de pacientes asintomáticos; las imágenes serán obtenidas del archivo del CDI, previa solicitud al director. Las imágenes TCHC serán analizadas con el software RealScan 2.0 (Point Nix. Corea) para los defectos óseos mediante reconstrucción multiplanar en vistas axiales, coronales, sagitales, cortes transaxiales, así como la evaluación mediante reconstrucciones $3 \mathrm{D}$ en volumen rendering y para la inclinación de la eminencia articular, una de las vistas axiales sobre la cual se observan los procesos condilares en su más amplia extensión lateral medial y que es utilizada como vista de referencia para la reconstrucción secundaria. Resultados: El promedio de edad fue de 30 años \pm 6,13 y no hubo diferencias significativas entre sexos. El ángulo ANB en promedio la muestra fue de $7,02^{\circ} \pm 1,80^{\circ}$ y no hubo diferencias significativas entre géneros. Los valores cuantitativos de la inclinación de la eminencia articular medidos a través de los ángulos EBT y ETR no tuvieron diferencias significativas entre lados. No se encontró asociación significativa entre el ángulo de la eminencia articular EBT y la presencia de cambios óseos degenerativos. En el caso de la asociación entre el grado del ángulo de la eminencia articular ETR y la presencia de cambios óseos degenerativos, el aplanamiento mostró del lado izquierdo y se encontró asociación significativa con la erosión en el lado derecho. Conclusiones: La inclinación de la eminencia articular medida en el ángulo EBT de pacientes clase II esquelética y su relación con cambios osteoatrósicos según lado no muestra asociaciones significativas. La inclinación de la eminencia articular medida en el ángulo ETR de pacientes clase II

Citar como: Pardo-Juárez V. Relación entre la presencia de cambios óseos de osteoartrosis del cóndilo mandibular e inclinación de la eminencia articular en pacientes clase II esquelética evaluados mediante tomografía computarizada de haz cónico. Rev Cient Odontol (Lima). 2019;7 (1): 34-52. 
esquelética y su relación con cambios osteoartrósicos, según lado del aplanamiento, mostró una asociación significativa en lado izquierdo y una asociación significativa en la erosión del lado derecho.

Palabras clave: osteoartritis, osteoartrosis, cambios óseos degenerativos, eminencia articular, clase II esqueletal, osteofito, aplanamiento

\section{ABSTRACT}

Objetive: Identify the relationship between the inclination of the articular eminence of skeletal class II patients with signs of osteoarthrosis measured by cone beam computed tomography (CBCT) in patients from the Diagnostic Imaging Center (CDI) in Lima, Perú between 2010 and 2014. Material and method: We performed a retrospective study of CBCT explorations in asymptomatic patients. The images were obtained from the head of the Radiology Department of the CDI in Lima, Peru. The CBCT images were analyzed with RealScan 2.0 software (Point Nix. Korea) for bone defects by multiplanar reconstruction in axial, coronal, sagittal, transaxial planes as well as evaluation by $3 \mathrm{D}$ reconstruction in volume rendering and for the inclination of the articular eminence. An axial view showing the condylar processes at their broadest lateral medial extension was used as the reference for secondary reconstruction. Results: The mean age of the patients studied was 30 years \pm 6.13 , with no significant differences between sexes. The mean ANB angle was $7.02^{\circ}$ $\pm 1.80^{\circ}$, with no significant differences between sexes. The quantitative values of the inclination of the joint eminence measured according to the EBT and ETR angles, showed no significant differences between sides There was no significant association between the degree of the angle of the EBT articular eminence and the presence of degenerative bone changes. The association between the degree of the ETR joint eminence shown by flattening on the left side and the presence of degenerative bone changes demonstrated by erosion on the right side was found to be significant. Coclusions: No significant association was found between the inclination of the joint eminence measured in the EBT angle of skeletal class II patients and its relationship with osteoarthritic changes. There was a significant association between the inclination of the joint eminence at the ETR angles shown by flattening on the left side and osteoarthritic changes observed as erosion on the right side.

Keywords: osteoarthrosis, degenerative bone changes, joint eminence, skeletal class II, osteophyte, flattening

\section{INTRODUCCIÓN}

La inclinación de la eminencia articular puede variar entre los individuos y determina la trayectoria de movimiento condíleo, así como el grado de rotación del disco sobre el cóndilo $\left(^{1-10}\right)$. La profundidad de la cavidad glenoidea varía y el desarrollo de la eminencia articular depende de los estímulos funcionales que tenga el cóndilo $\left.{ }^{11-20}\right)$. Estudios sugieren que la formación ósea de compensación de la cavidad glenoidea podría ayudar a soportar el aumento de las sobrecargas en la ATM que acompañan los cambios óseos degenerativos condilares $(2,11-15)$.

Tomando esto en cuenta, puede deducirse que la eminencia articular se desarrolla casi totalmente después del nacimiento. El hecho de que la ausencia condilar congénita esté acompañada por un subdesarrollo de la eminencia articular demuestra una fuerte dependencia funcional mutua entre el cóndilo y la cavidad glenoidea. Una opinión que 
se respalda por estos estudios es que el desarrollo de la eminencia articular y el cóndilo mandibular se ve más afectado por la función que por las características de la base del cráneo $\left({ }^{15-19}\right)$.

La TCHC proporciona imágenes con resolución submilimétrica de muy alta calidad, lo cual hace que el diagnóstico de la ATM sea preciso $\left({ }^{19-30}\right)$. Anteriormente, se ha utilizado la TCHC para evaluar la inclinación de la eminencia articular y la morfología condilar, pues proporciona mediciones lineales precisas y fiables para la reconstrucción y la obtención de imágenes de alta resolución de la región maxilofacial; además, es una modalidad de imagen que trabaja con una dosis de radiación efectiva mucho menor en comparación con la tomografía computarizada multicorte $\left({ }^{30-35}\right)$. En este caso, la TCHC ayudará en este trabajo el evaluar la relación entre la presencia de cambios óseos de osteoartrosis del cóndilo mandibular y la inclinación de la eminencia articular en pacientes clase II esquelética.

\section{MATERIALES Y MÉTODOS}

El grupo de estudio retrospectivo fue planificado de acuerdo al tamaño de muestra de estimación y consistió en exploraciones con TCHC de pacientes asintomáticos. Las imágenes fueron obtenidas del archivo del Departamento de Radiología del CDI (Lima, Perú), previa solicitud al director. Para ello, se solicitó un permiso al director del CDI.

Según el reporte del CDI, las imágenes de TCHC fueron tomadas usando el equipo Vatech E-woo modelo Picasso master de origen coreano, operado a $90 \mathrm{kVp}$ y $8 \mathrm{~mA}$, con un tiempo de exposición de 20 segundos y un tamaño de voxel de $0,4 \mathrm{~mm}$. Todas las imágenes se adquieren siguiendo el protocolo recomendado de acuerdo a los requeri- mientos de este tipo de estudios, se usa un FOV de 20 x $19 \mathrm{~cm}$.

Las imágenes TCHC fueron analizadas con el software RealScan 2.0 (Point Nix. Corea). Para los defectos óseos mediante reconstrucción multiplanar en vistas axiales, coronales, sagitales, cortes transaxiales, así como la evaluación mediante reconstrucciones $3 \mathrm{D}$ en volumen rendering y para la inclinación de la eminencia articular, una de las vistas axiales sobre la cual los se observan los procesos condilares en su más amplia extensión lateral medial siendo utilizada como vista de referencia para la reconstrucción secundaria.

Las reconstrucciones en $3 D$ fueron creadas reformateando los escáneres de TCHC axiales en una estación de trabajo local utilizando el mismo software.

La manipulación del volumen se realizó en una estación de trabajo Samsung Intel Core i7-4770, visualizadas en un monitor S19C150 Samsung LCD con retroiluminación LED de 18.5 pulgadas, pantalla panorámica, con una resolución de 1366 x 768 píxeles en un ambiente con luz tenue.

El contraste y brillo de las imágenes fueron ajustados usando la herramienta procesadora de imágenes en el software para asegurar una visualización óptima.

Un consentimiento informado escrito se firmó y que se obtiene habitualmente de cada paciente antes del estudio.

Se realizó la prueba piloto a través de la evaluación independiente y calibrada con 10 unidades de estudio asesorado por un radiólogo bucal y maxilofacial. Para la calibración se usó el estadístico índice de kappa, permitiendo calcular la concordancia interexaminador e intraexaminador. 
Antes de que se realizaran las mediciones, se determinó la orientación de las imágenes tomográficas para cada paciente y se utilizó el plano de Frankfort; se llevó a cabo en paralelo al plano horizontal en la vista lateral.

Los ángulos utilizados para determinar la inclinación de la eminencia articular fueron: 1. Mediante el ángulo entre EBT y Frankfort horizontal. Este ángulo está formado por el plano EBT que es el plano de mejor ajuste de la inclinación eminencia articular y el plano de Frankfort. Para el ángulo EBT, se utiliza el plano EBT, es el plano de mejor ajuste de la inclinación de la eminencia articular. El plano de Frankfort: es el plano que va de Po a Or; Po: Pórion. Es el punto más alto del conducto auditivo externo; Or: Es el punto ubicado en borde inferior del reborde orbitario. 2. Mediante el ángulo Etr y Frankfort horizontal. Este ángulo está formado por Plano Etr que es el plano que pasa por los puntos de $\mathrm{Cu}$ y $\mathrm{R}$ y el plano de Frankfort; Para el ángulo Etr se utilizan los puntos y planos: Plano Etr: es el plano que pasa por los puntos de $\mathrm{Cu}$ y $\mathrm{R}$; $\mathrm{Cu}$ : el punto más bajo de la eminencia articular; R: Es el punto más alto de la cavidad glenoidea; El Plano de Frankfort explicado anteriormente.

Los cóndilos se clasificaron y se agruparon con cambios óseos y sin cambios óseos; Los cambios óseos se agruparon en sin osteoartritis; con aplanamiento; con osteofíto; con erosión; con esclerosis para su clasificación el plano coronal se colocó paralelo al eje longitudinal del cóndilo y el plano sagital se fijó perpendicularmente al coronal; En el plano sagital todos los datos se registraron en ambos lados.

\section{RESULTADOS}

En la tabla 1 se describen las características iniciales de la muestra. De un total de 50 pacientes 21 eran hombres y 29 eran mujeres, el promedio de edad de la muestra completa fue de 30 años \pm 6.13 no habiendo diferencias significativas entre sexos. El ángulo ANB en promedio para toda la muestra fue de $7.02^{\circ} \pm 1.80^{\circ}$ tampoco hubo diferencias significativas entre géneros.

En la tabla 2 se aprecian los valores cuantitativos de la inclinación de la eminencia articular medidos a través de los ángulos EBT Y ETR, no habiendo diferencias significativas entre lados.

En la tabla 3 se observa la prevalencia de cambio óseos de osteoartrosis según lado, no observándose diferencias significativas entre lados. La prevalencia de osteofitos fue de $20 \%$, del aplanamiento condilar $82 \%$, de la erosión fluctuó entre $50 \%$ y $56 \%$, la de esclerosis subcondral varió entre $46 \%$ y $72 \%$, la de quiste subcondral varió entre $14 \%$ y $16 \%$ y no hubo ningún caso que presentó cuerpos libres en ambos lados.

La tabla 4 muestra la asociación entre el grado del ángulo de la eminencia articular EBT y la presencia de osteofitos en cada lado. El ángulo EBT fue categorizado en tres grupos, bajo de $0^{\circ}$ a $30^{\circ}$, moderado de $30^{\circ}$ a $60^{\circ}$ y alto de $60^{\circ}$ a $90^{\circ}$, y no se encontró asociación significativa entre alguna de estas categorías y la presencia de osteofitos en ambos lados.

La tabla 5 muestra la asociación entre el grado de ángulo EBT y la presencia del aplanamiento por lado, no observándose la influencia de alguna categoría del ángulo con la presencia del aplanamiento condilar.

La erosión condilar, la esclerosis subcondral, la presencia del quiste subcondral, tampoco estuvieron asociadas a alguna categoría del ángulo EBT, (Tabla 6, 7 y 8.) 
En la tabla 9 se observa que en ninguna categoría del ángulo EBT se presentaron cuerpos libres en ambos cóndilos.

En la tabla 10 se observa la asociación entre el grado del ángulo ETR y la presencia de osteofitos en cada lado, no se observa asociación significativa entre ambos lados.

En la tabla 11 se asocia el grado del ángulo ETR y la presencia de aplanamiento, y se observa una asociación significativa en el lado izquierdo, con la categoría de ángulo bajo asociada a la presencia de aplanamiento en el $100 \%$ de los casos. La categoría de ángulo moderado presentó un $75 \%$ de cóndilos con aplanamiento y un $25 \%$ con ausencia de aplanamiento. Sin embargo, para darle un valor clínico a este resultado, el hallazgo debió mostrarse en ambos cóndilos, lo que no fue observado.
En la tabla 12 se observa la asociación entre el ángulo ETR agrupado y la presencia de erosión, y se encontró asociación significativa en el lado derecho. Los pacientes con ángulo bajo presentaron erosión en el $83 \%$ de casos, mientras que en el ángulo moderado la presentó el $30.80 \%$. Igualmente, este resultado no se observó en el lado izquierdo, situación que debe ser analizada desde el punto de vista clínico.

En las tablas 13 y 14 no se observan asociaciones significativas entre las categorías del ángulo ETR y la presencia de la esclerosis subcondral o del quiste subcondral en ambos cóndilos.

Finalmente, en la tabla 15 se observa que ninguna categoría del ángulo ETR presentó cuerpos libres en ambos cóndilos.

Tabla 1. Características iniciales de la muestra de acuerdo con la edad y al ángulo ANB, SEGÚN SEXO

\begin{tabular}{|l|c|c|c|c|c|c|}
\hline \multirow{2}{*}{ Sexo } & \multirow{2}{*}{${ }^{\circ}$} & $\%$ & \multicolumn{2}{|c|}{ Edad } & \multicolumn{3}{|c|}{ Ángulo ANB } \\
\hline Masculino & 21 & 42 & 29,62 & 6,36 & 7,13 & 2,03 \\
\hline Femenino & 29 & 58 & 30,28 & 6,06 & 6,95 & 1,66 \\
\hline Total & 50 & 100 & 30,00 & 6,13 & 7,02 & 1,80 \\
\hline
\end{tabular}

$p>0,05$ en edad y ANB

Prueba T de Student

TABLA 2. InCLINACIÓN DE LA EMINENCIA ARTICULAR (ÁNGULOS EBT y ETR) EN LOS PACIENTES EVALUAdOS

\begin{tabular}{|l|c|c|c|c|c|}
\hline Mediciones & n. $^{\circ}$ & Media & D. E. & Mínimo & Máximo \\
\hline Ángulo EBT lado derecho & 50 & 54,66 & 13,83 & 27,00 & 80,20 \\
\hline Ángulo EBT lado izquierdo & 50 & 54,85 & 13,61 & 28,30 & 89,10 \\
\hline Ángulo ETR lado derecho & 50 & 31,70 & 6,98 & 20,70 & 47,00 \\
\hline Ángulo ETR lado izquierdo & 50 & 33,20 & 6,48 & 22,90 & 50,60 \\
\hline
\end{tabular}

$p>0,05$ entre lados

Prueba T de Student pareada 
Relación entre la presencia de cambios óseos de osteoartrosis del cóndilo mandibular e inclinación de la eminencia articular en pacientes clase II esquelética evaluados mediante tomografía computarizada de haz cónico

Tabla 3. Prevalencia de cambios óseos de osteoartrosis en el Cóndilo mandibular, Según lado en LOS PACIENTES EVALUADOS

\begin{tabular}{|c|c|c|c|c|c|c|c|}
\hline \multirow{2}{*}{ Lado } & \multirow{2}{*}{ Patología } & \multicolumn{2}{|c|}{ Presente } & \multicolumn{2}{|c|}{ Ausente } & \multicolumn{2}{|c|}{ Total } \\
\hline & & $\mathrm{n} .^{\circ}$ & $\%$ & n. & $\%$ & $\mathrm{n}^{\circ}$ & $\%$ \\
\hline \multirow{6}{*}{ Derecho } & Osteofitos & 10 & 20 & 40 & 80 & 50 & 100 \\
\hline & Aplanamiento & 41 & 82 & 9 & 18 & 50 & 100 \\
\hline & Erosión & 28 & 56 & 22 & 44 & 50 & 100 \\
\hline & Esclerosis subcondral & 36 & 72 & 14 & 28 & 50 & 100 \\
\hline & Quiste subcondral & 7 & 14 & 43 & 86 & 50 & 100 \\
\hline & Cuerpos libres & 0 & 0 & 50 & 100 & 50 & 100 \\
\hline \multirow{6}{*}{ Izquierdo } & Osteofitos & 10 & 20 & 40 & 80 & 50 & 100 \\
\hline & Aplanamiento & 41 & 82 & 9 & 18 & 50 & 100 \\
\hline & Erosión & 25 & 50 & 25 & 50 & 50 & 100 \\
\hline & Esclerosis subcondral & 23 & 46 & 27 & 54 & 50 & 100 \\
\hline & Quiste subcondral & 8 & 16 & 42 & 84 & 50 & 100 \\
\hline & Cuerpos libres & 0 & 0 & 50 & 100 & 50 & 100 \\
\hline
\end{tabular}

$p>0,05$ entre lados

Prueba de chi cuadrado

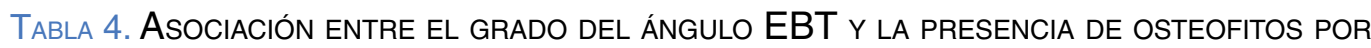
LADO

\begin{tabular}{|c|c|c|c|c|c|c|c|}
\hline \multirow{2}{*}{ Lado } & \multirow{2}{*}{ Medida } & \multirow{2}{*}{\multicolumn{2}{|c|}{ Categorías }} & \multicolumn{2}{|c|}{ Osteofitos } & \multirow{2}{*}{ Total } & \multirow{2}{*}{$\mathrm{p}$} \\
\hline & & & & Ausente & Presente & & \\
\hline \multirow{6}{*}{ Derecho } & \multirow{6}{*}{ Ángulo EBT } & \multirow{2}{*}{ Alto $\left(60^{\circ}\right.$ a $\left.90^{\circ}\right)$} & n. ${ }^{\circ}$ & 14 & 4 & 18 & \multirow{6}{*}{0,579} \\
\hline & & & $\%$ & $77,78 \%$ & $22,22 \%$ & $100,00 \%$ & \\
\hline & & \multirow{2}{*}{ Moderado $\left(30^{\circ}\right.$ a $\left.60^{\circ}\right)$} & n. ${ }^{\circ}$ & 22 & 6 & 28 & \\
\hline & & & $\%$ & $78,57 \%$ & $21,43 \%$ & $100,00 \%$ & \\
\hline & & \multirow{2}{*}{ Bajo $\left(0^{\circ}\right.$ a $\left.30^{\circ}\right)$} & $\mathrm{n} .{ }^{\circ}$ & 4 & 0 & 4 & \\
\hline & & & $\%$ & $100,00 \%$ & $0,00 \%$ & $100,00 \%$ & \\
\hline \multirow{6}{*}{ Izquierdo } & \multirow{6}{*}{ Ángulo EBT } & \multirow{2}{*}{ Alto $\left(60\right.$ a $\left.90^{\circ}\right)$} & n. ${ }^{\circ}$ & 11 & 4 & 15 & \multirow{6}{*}{0,611} \\
\hline & & & $\%$ & $73,30 \%$ & $26,70 \%$ & $100,00 \%$ & \\
\hline & & \multirow{2}{*}{ Moderado $\left(30^{\circ}\right.$ a $\left.60^{\circ}\right)$} & n. ${ }^{\circ}$ & 27 & 6 & 33 & \\
\hline & & & $\%$ & $81,80 \%$ & $18,20 \%$ & $100,00 \%$ & \\
\hline & & \multirow{2}{*}{ Bajo $\left(0^{\circ}\right.$ a $\left.30^{\circ}\right)$} & n. ${ }^{\circ}$ & 2 & 0 & 2 & \\
\hline & & & $\%$ & 100,00 & 0,00 & $100,00 \%$ & \\
\hline
\end{tabular}

Prueba de chi cuadrado 
TABLA 5. Asociación ENTRE EL GRAdo del ÁNGULO EBT Y LA PRESENCIA DE APLANAMIENTO POR LADO

\begin{tabular}{|c|c|c|c|c|c|c|c|}
\hline \multirow{2}{*}{ Lado } & \multirow{2}{*}{ Medida } & \multirow{2}{*}{\multicolumn{2}{|c|}{ Categorías }} & \multicolumn{2}{|c|}{ Aplanamiento } & \multirow{2}{*}{ Total } & \multirow{2}{*}{$\mathrm{p}$} \\
\hline & & & & Ausente & Presente & & \\
\hline \multirow{6}{*}{ Derecho } & \multirow{6}{*}{ Ángulo EBT } & \multirow{2}{*}{ Alto $\left(60^{\circ}\right.$ a $\left.90^{\circ}\right)$} & n. & 4 & 14 & 18 & \multirow{6}{*}{0,736} \\
\hline & & & $\%$ & $22,20 \%$ & $77,80 \%$ & $100,00 \%$ & \\
\hline & & \multirow{2}{*}{ Moderado $\left(30^{\circ}\right.$ a $\left.60^{\circ}\right)$} & n. ${ }^{\circ}$ & 4 & 24 & 28 & \\
\hline & & & $\%$ & $14,3 \%$ & $85,7 \%$ & $100,00 \%$ & \\
\hline & & \multirow{2}{*}{ Bajo $\left(0^{\circ}\right.$ a $\left.30^{\circ}\right)$} & n. ${ }^{\circ}$ & 1 & 3 & 4 & \\
\hline & & & $\%$ & $25,00 \%$ & $75,00 \%$ & $100,00 \%$ & \\
\hline \multirow{6}{*}{ Izquierdo } & \multirow{6}{*}{ Ángulo EBT } & \multirow{2}{*}{ Alto $\left(60^{\circ}\right.$ a $\left.90^{\circ}\right)$} & n. ${ }^{\circ}$ & 4 & 11 & 15 & \multirow{6}{*}{0,501} \\
\hline & & & $\%$ & $26,70 \%$ & $73,30 \%$ & $100,00 \%$ & \\
\hline & & \multirow{2}{*}{ Moderado $\left(30^{\circ}\right.$ a $\left.60^{\circ}\right)$} & n. & 5 & 28 & 33 & \\
\hline & & & $\%$ & $15,20 \%$ & $84,80 \%$ & $100,00 \%$ & \\
\hline & & \multirow{2}{*}{ Bajo $\left(0^{\circ}\right.$ a $\left.30^{\circ}\right)$} & n. ${ }^{\circ}$ & 0 & 2 & 2 & \\
\hline & & & $\%$ & 0,00 & 100,00 & $100,00 \%$ & \\
\hline
\end{tabular}

Prueba de chi cuadrado

TABLA 6. Asociación ENTRE El GRAdo del Ángulo EBT y LA PRESENCIA de EROSIÓN POR LADO

\begin{tabular}{|c|c|c|c|c|c|c|c|}
\hline \multirow{2}{*}{ Lado } & \multirow{2}{*}{ Medida } & \multirow{2}{*}{\multicolumn{2}{|c|}{ Categorias }} & \multicolumn{2}{|c|}{ Erosión } & \multirow{2}{*}{ Total } & \multirow{2}{*}{$p$} \\
\hline & & & & Ausente & Presente & & \\
\hline \multirow{6}{*}{ Derecho } & \multirow{6}{*}{ Ángulo EBT } & \multirow{2}{*}{ Alto $\left(60^{\circ}\right.$ a $\left.90^{\circ}\right)$} & n. ${ }^{\circ}$ & 11 & 7 & 18 & \multirow{6}{*}{0,063} \\
\hline & & & $\%$ & $61,10 \%$ & $38,90 \%$ & $100,00 \%$ & \\
\hline & & \multirow{2}{*}{$\begin{array}{c}\text { Moderado }\left(30^{\circ} \mathrm{a}\right. \\
\left.60^{\circ}\right)\end{array}$} & $\mathrm{n} .^{\circ}$ & 11 & 17 & 28 & \\
\hline & & & $\%$ & $39,30 \%$ & $60,70 \%$ & $100,00 \%$ & \\
\hline & & \multirow{2}{*}{ Bajo $\left(0^{\circ}\right.$ a $\left.30^{\circ}\right)$} & n. & 0 & 4 & 4 & \\
\hline & & & $\%$ & $0,00 \%$ & $100,00 \%$ & $100,00 \%$ & \\
\hline \multirow{6}{*}{ Izquierdo } & \multirow{6}{*}{ Ángulo EBT } & \multirow{2}{*}{ Alto $\left(60^{\circ}\right.$ a $\left.90^{\circ}\right)$} & $\mathrm{n} .^{\circ}$ & 7 & 8 & 15 & \multirow{6}{*}{0,305} \\
\hline & & & $\%$ & $46,70 \%$ & $53,30 \%$ & $100,00 \%$ & \\
\hline & & \multirow{2}{*}{$\begin{array}{c}\text { Moderado }\left(30^{\circ} \mathrm{a}\right. \\
\left.60^{\circ}\right)\end{array}$} & n. ${ }^{\circ}$ & 16 & 17 & 33 & \\
\hline & & & $\%$ & $48,50 \%$ & $51,50 \%$ & $100,00 \%$ & \\
\hline & & \multirow{2}{*}{ Bajo $\left(0^{\circ}\right.$ a $\left.30^{\circ}\right)$} & $\mathrm{n}$. & 2 & 0 & 2 & \\
\hline & & & $\%$ & 100,00 & 0,00 & $100,00 \%$ & \\
\hline
\end{tabular}

Prueba de chi cuadrado 
Relación entre la presencia de cambios óseos de osteoartrosis del cóndilo mandibular e inclinación de la eminencia articular en pacientes clase II esquelética evaluados mediante tomografía computarizada de haz cónico

Tabla 7. Asociación entre el GRAdo del Ángulo EBT y la PRESENCIA de ESCLEROSIS SUBCONDRAL POR LADO

\begin{tabular}{|c|c|c|c|c|c|c|c|}
\hline \multirow{2}{*}{ Lado } & \multirow{2}{*}{ Medida } & \multirow{2}{*}{\multicolumn{2}{|c|}{ Categorías }} & \multicolumn{2}{|c|}{ Esclerosis subcondral } & \multirow{2}{*}{ Total } & \multirow{2}{*}{$p$} \\
\hline & & & & Ausente & Presente & & \\
\hline \multirow{6}{*}{ Derecho } & \multirow{6}{*}{ Ángulo EBT } & \multirow{2}{*}{ Alto $\left(60^{\circ}\right.$ a $\left.90^{\circ}\right)$} & n. ${ }^{\circ}$ & 14 & 4 & 18 & \multirow{6}{*}{0,262} \\
\hline & & & $\%$ & $77,80 \%$ & $22,20 \%$ & $100,00 \%$ & \\
\hline & & \multirow{2}{*}{$\begin{array}{c}\text { Moderado }\left(30^{\circ} \mathrm{a}\right. \\
\left.60^{\circ}\right)\end{array}$} & n. ${ }^{\circ}$ & 18 & 10 & 28 & \\
\hline & & & $\%$ & $64,30 \%$ & $35,70 \%$ & $100,00 \%$ & \\
\hline & & \multirow{2}{*}{ Bajo $\left(0^{\circ}\right.$ a $\left.30^{\circ}\right)$} & n. ${ }^{\circ}$ & 4 & 0 & 4 & \\
\hline & & & $\%$ & $100,00 \%$ & $0,00 \%$ & $100,00 \%$ & \\
\hline \multirow{6}{*}{ Izquierdo } & \multirow{6}{*}{ Ángulo EBT } & \multirow{2}{*}{ Alto $\left(60^{\circ}\right.$ a $\left.90^{\circ}\right)$} & n. ${ }^{\circ}$ & 9 & 6 & 15 & \multirow{6}{*}{0,855} \\
\hline & & & $\%$ & $60,00 \%$ & $40,00 \%$ & $100,00 \%$ & \\
\hline & & \multirow{2}{*}{$\begin{array}{c}\text { Moderado }\left(30^{\circ} \mathrm{a}\right. \\
\left.60^{\circ}\right)\end{array}$} & n. ${ }^{\circ}$ & 17 & 16 & 33 & \\
\hline & & & $\%$ & $51,50 \%$ & $48,50 \%$ & $100,00 \%$ & \\
\hline & & \multirow{2}{*}{ Bajo $\left(0^{\circ}\right.$ a $\left.30^{\circ}\right)$} & n. ${ }^{\circ}$ & 1 & 1 & 2 & \\
\hline & & & $\%$ & 50,00 & 50,00 & $100,00 \%$ & \\
\hline
\end{tabular}

Prueba de chi cuadrado

TABLA 8. AsociACIÓN ENTRE EL GRADO DEL ÁNGULO EBT Y LA PRESENCIA DE QUISTE SUBCONDRAL POR LADO

\begin{tabular}{|c|c|c|c|c|c|c|c|}
\hline \multirow{2}{*}{ Lado } & \multirow{2}{*}{ Medida } & \multirow{2}{*}{\multicolumn{2}{|c|}{ Categorías }} & \multicolumn{2}{|c|}{ Quiste subcondral } & \multirow{2}{*}{ Total } & \multirow{2}{*}{$\mathrm{p}$} \\
\hline & & & & Ausente & Presente & & \\
\hline \multirow{6}{*}{ Derecho } & \multirow{6}{*}{ Ángulo EBT } & \multirow{2}{*}{ Alto $\left(60^{\circ}\right.$ a $\left.90^{\circ}\right)$} & n. & 17 & 1 & 18 & \multirow{6}{*}{0,404} \\
\hline & & & $\%$ & $94,40 \%$ & $5,60 \%$ & $100,00 \%$ & \\
\hline & & \multirow{2}{*}{ Moderado $\left(30^{\circ}\right.$ a $\left.60^{\circ}\right)$} & n. ${ }^{\circ}$ & 23 & 5 & 28 & \\
\hline & & & $\%$ & $82,10 \%$ & $17,90 \%$ & $100,00 \%$ & \\
\hline & & \multirow{2}{*}{ Bajo $\left(0^{\circ}\right.$ a $\left.30^{\circ}\right)$} & n. & 3 & 1 & 4 & \\
\hline & & & $\%$ & $75,00 \%$ & $25,00 \%$ & $100,00 \%$ & \\
\hline \multirow{6}{*}{ Izquierdo } & \multirow{6}{*}{ Ángulo EBT } & \multirow{2}{*}{ Alto $\left(60^{\circ}\right.$ a $\left.90^{\circ}\right)$} & n. ${ }^{\circ}$ & 14 & 1 & 15 & \multirow{6}{*}{0,364} \\
\hline & & & $\%$ & $93,30 \%$ & $6,70 \%$ & $100,00 \%$ & \\
\hline & & \multirow{2}{*}{ Moderado $\left(30^{\circ}\right.$ a $\left.60^{\circ}\right)$} & n. ${ }^{\circ}$ & 26 & 7 & 33 & \\
\hline & & & $\%$ & $78,80 \%$ & $21,20 \%$ & $100,00 \%$ & \\
\hline & & \multirow{2}{*}{ Bajo $\left(0^{\circ}\right.$ a $\left.30^{\circ}\right)$} & n. ${ }^{\circ}$ & 2 & 0 & 2 & \\
\hline & & & $\%$ & 100,00 & 0,00 & $100,00 \%$ & \\
\hline
\end{tabular}

Prueba de chi cuadrado 
TABLA 9. Asociación ENTRE EL GRADO del ÁNGULO EBT y LA PRESENCIA DE CUERPOS LIBRES POR LADO

\begin{tabular}{|c|c|c|c|c|c|c|c|}
\hline \multirow{2}{*}{ Lado } & \multirow{2}{*}{ Medida } & \multirow{2}{*}{\multicolumn{2}{|c|}{ Categorías }} & \multicolumn{2}{|c|}{ Cuerpos libres } & \multirow{2}{*}{ Total } & \multirow{2}{*}{$\mathrm{p}$} \\
\hline & & & & Ausente & Presente & & \\
\hline \multirow{6}{*}{ Derecho } & \multirow{6}{*}{ Ángulo EBT } & \multirow{2}{*}{ Alto $\left(60^{\circ}\right.$ a $\left.90^{\circ}\right)$} & n. ${ }^{\circ}$ & 18 & 0 & 18 & \multirow{6}{*}{ no } \\
\hline & & & $\%$ & $100,00 \%$ & $0,00 \%$ & $100,00 \%$ & \\
\hline & & \multirow{2}{*}{ Moderado $\left(30^{\circ}\right.$ a $\left.60^{\circ}\right)$} & n. ${ }^{\circ}$ & 28 & 0 & 28 & \\
\hline & & & $\%$ & $100,00 \%$ & $0,00 \%$ & $100,00 \%$ & \\
\hline & & \multirow{2}{*}{ Bajo $\left(0^{\circ}\right.$ a $\left.30^{\circ}\right)$} & n. & 4 & 0 & 4 & \\
\hline & & & $\%$ & $100,00 \%$ & $0,00 \%$ & $100,00 \%$ & \\
\hline \multirow{6}{*}{ Izquierdo } & \multirow{6}{*}{ Ángulo EBT } & \multirow{2}{*}{ Alto $\left(60^{\circ}\right.$ a $\left.90^{\circ}\right)$} & $\mathrm{n} .^{\circ}$ & 15 & 0 & 15 & \multirow{6}{*}{ nc } \\
\hline & & & $\%$ & $100,00 \%$ & $0,00 \%$ & $100,00 \%$ & \\
\hline & & \multirow{2}{*}{ Moderado $\left(30^{\circ}\right.$ a $\left.60^{\circ}\right)$} & $\mathrm{n} .^{\circ}$ & 33 & 0 & 33 & \\
\hline & & & $\%$ & $100,00 \%$ & $0,00 \%$ & $100,00 \%$ & \\
\hline & & \multirow{2}{*}{ Bajo $\left(0^{\circ}\right.$ a $\left.30^{\circ}\right)$} & n. ${ }^{\circ}$ & 2 & 0 & 2 & \\
\hline & & & $\%$ & $100,00 \%$ & $0,00 \%$ & $100,00 \%$ & \\
\hline
\end{tabular}

$\mathrm{nc}=$ no calculable

TABLA 10. AsOCIACIÓN ENTRE EL GRADO DEL ÁNGULO ETR Y LA PRESENCIA DE OSTEOFITOS POR LADO

\begin{tabular}{|c|c|c|c|c|c|c|c|}
\hline \multirow{2}{*}{ Lado } & \multirow{2}{*}{ Medida } & \multirow{2}{*}{\multicolumn{2}{|c|}{ Categorías }} & \multicolumn{2}{|c|}{ Osteofitos } & \multirow{2}{*}{ Total } & \multirow{2}{*}{$p$} \\
\hline & & & & Ausente & Presente & & \\
\hline \multirow{6}{*}{ Derecho } & \multirow{6}{*}{ Ángulo ETR } & \multirow{2}{*}{ Alto $\left(60^{\circ}\right.$ a $\left.90^{\circ}\right)$} & $\mathrm{n}$ & 0 & 0 & 0 & \multirow{6}{*}{0,203} \\
\hline & & & $\%$ & $0,00 \%$ & $0,00 \%$ & $0,00 \%$ & \\
\hline & & \multirow{2}{*}{ Moderado $\left(30^{\circ}\right.$ a $\left.60^{\circ}\right)$} & $\mathrm{n}$ & 19 & 7 & 26 & \\
\hline & & & $\%$ & $73,10 \%$ & $26,90 \%$ & $100,00 \%$ & \\
\hline & & \multirow{2}{*}{ Bajo $\left(0^{\circ}\right.$ a $\left.30^{\circ}\right)$} & $\mathrm{n}$ & 21 & 3 & 24 & \\
\hline & & & $\%$ & $87,50 \%$ & $12,50 \%$ & $100,00 \%$ & \\
\hline \multirow{6}{*}{ Izquierdo } & \multirow{6}{*}{ Ángulo ETR } & \multirow{2}{*}{ Alto $\left(60^{\circ}\right.$ a $\left.90^{\circ}\right)$} & $\mathrm{n}$ & 0 & 0 & 0 & \multirow{6}{*}{0,156} \\
\hline & & & $\%$ & $0,00 \%$ & $0,00 \%$ & $0,00 \%$ & \\
\hline & & \multirow{2}{*}{ Moderado $\left(30^{\circ}\right.$ a $\left.60^{\circ}\right)$} & $\mathrm{n}$ & 27 & 9 & 36 & \\
\hline & & & $\%$ & $75,00 \%$ & $25,00 \%$ & $100,00 \%$ & \\
\hline & & \multirow{2}{*}{ Bajo $\left(0^{\circ}\right.$ a $\left.30^{\circ}\right)$} & $\mathrm{n}$ & 13 & 1 & 2 & \\
\hline & & & $\%$ & 92,90 & 7,10 & $100,00 \%$ & \\
\hline
\end{tabular}

Prueba de chi cuadrado 
Relación entre la presencia de cambios óseos de osteoartrosis del cóndilo mandibular e inclinación de la eminencia articular en pacientes clase II esquelética evaluados mediante tomografía computarizada de haz cónico

TABLA 11. Asociación ENTRE EL GRADO del ÁNGULO ETR Y LA PRESENCIA DE APLANAMIENTO POR LADO

\begin{tabular}{|c|c|c|c|c|c|c|c|}
\hline I ado & & \multirow{2}{*}{\multicolumn{2}{|c|}{ Categorías }} & \multicolumn{2}{|c|}{ Aplanamiento } & \multirow{2}{*}{ Total } & \multirow{2}{*}{$p$} \\
\hline Lado & IVlealaa & & & Ausente & Presente & & \\
\hline \multirow{6}{*}{ Derecho } & \multirow{6}{*}{ Ángulo ETR } & \multirow{2}{*}{ Alto $\left(60^{\circ}\right.$ a $\left.90^{\circ}\right)$} & n. ${ }^{\circ}$ & 0 & 0 & 0 & \multirow{6}{*}{0,331} \\
\hline & & & $\%$ & $0,00 \%$ & $0,00 \%$ & $0,00 \%$ & \\
\hline & & \multirow{2}{*}{$\begin{array}{c}\text { Moderado }\left(30^{\circ} \mathrm{a}\right. \\
\left.60^{\circ}\right)\end{array}$} & n. ${ }^{\circ}$ & 6 & 20 & 26 & \\
\hline & & & $\%$ & $23,10 \%$ & $76,90 \%$ & $100,00 \%$ & \\
\hline & & \multirow{2}{*}{ Bajo $\left(0^{\circ}\right.$ a $\left.30^{\circ}\right)$} & n. ${ }^{\circ}$ & 3 & 21 & 24 & \\
\hline & & & $\%$ & $12,50 \%$ & $87,50 \%$ & $100,00 \%$ & \\
\hline \multirow{6}{*}{ Izquierdo } & \multirow{6}{*}{ Ángulo ETR } & \multirow{2}{*}{ Alto $\left(60\right.$ a $\left.90^{\circ}\right)$} & n. ${ }^{\circ}$ & 0 & 0 & 0 & \multirow{6}{*}{$0,039^{*}$} \\
\hline & & & $\%$ & $0,00 \%$ & $0,00 \%$ & $0,00 \%$ & \\
\hline & & Moderado $\left(30^{\circ} \mathrm{a}\right.$ & n. ${ }^{\circ}$ & 9 & 27 & 36 & \\
\hline & & $\left.60^{\circ}\right)$ & $\%$ & $25,00 \%$ & $75,00 \%$ & $100,00 \%$ & \\
\hline & & \multirow{2}{*}{ Bajo $\left(0^{\circ}\right.$ a $\left.30^{\circ}\right)$} & n. & 0 & 14 & 14 & \\
\hline & & & $\%$ & 0,00 & 100,00 & $100,00 \%$ & \\
\hline
\end{tabular}

Prueba de chi cuadrado

*Significativo

TABLA 12. Asociación ENTRE el GRAdo del Ángulo ETR Y LA PRESENCIA dE EROSIÓN POR LADO

\begin{tabular}{|c|c|c|c|c|c|c|c|}
\hline \multirow{2}{*}{ Lado } & \multirow{2}{*}{ Medida } & \multirow{2}{*}{\multicolumn{2}{|c|}{ Categorías }} & \multicolumn{2}{|c|}{ Erosión } & \multirow{2}{*}{ Total } & \multirow{2}{*}{$\mathrm{p}$} \\
\hline & & & & Ausente & Presente & & \\
\hline \multirow{6}{*}{ Derecho } & \multirow{6}{*}{ Ángulo ETR } & \multirow{2}{*}{ Alto $\left(60^{\circ}\right.$ a $\left.90^{\circ}\right)$} & $\mathrm{n} .^{\circ}$ & 0 & 0 & 0 & \multirow{6}{*}{$<0,001^{*}$} \\
\hline & & & $\%$ & $0,00 \%$ & $0,00 \%$ & $0,00 \%$ & \\
\hline & & \multirow{2}{*}{ Moderado $\left(30^{\circ}\right.$ a $\left.60^{\circ}\right)$} & $\mathrm{n} .^{\circ}$ & 18 & 8 & 26 & \\
\hline & & & $\%$ & $69,20 \%$ & $30,80 \%$ & $100,00 \%$ & \\
\hline & & \multirow{2}{*}{ Bajo $\left(0^{\circ}\right.$ a $\left.30^{\circ}\right)$} & $\mathrm{n} .^{\circ}$ & 4 & 20 & 24 & \\
\hline & & & $\%$ & $16,70 \%$ & $83,30 \%$ & $100,00 \%$ & \\
\hline \multirow{6}{*}{ Izquierdo } & \multirow{6}{*}{ Ángulo ETR } & \multirow{2}{*}{ Alto $\left(60^{\circ}\right.$ a $\left.90^{\circ}\right)$} & $\mathrm{n} .^{\circ}$ & 0 & 0 & 0 & \multirow{6}{*}{0,529} \\
\hline & & & $\%$ & $0,00 \%$ & $0,00 \%$ & $0,00 \%$ & \\
\hline & & \multirow{2}{*}{ Moderado $\left(30^{\circ}\right.$ a $\left.60^{\circ}\right)$} & n. ${ }^{\circ}$ & 17 & 19 & 36 & \\
\hline & & & $\%$ & $47,20 \%$ & $52,80 \%$ & $100,00 \%$ & \\
\hline & & \multirow{2}{*}{ Bajo $\left(0^{\circ}\right.$ a $\left.30^{\circ}\right)$} & n. ${ }^{\circ}$ & 8 & 6 & 14 & \\
\hline & & & $\%$ & 57,10 & 42,90 & $100,00 \%$ & \\
\hline
\end{tabular}

Prueba de chi cuadrado

*Significativo 
TABLA 13. Asociación ENTRE EL GRADO DEL ÁNGULO ETR Y LA PRESENCIA DE ESCLEROSIS SUBCONDRAL POR LADO

\begin{tabular}{|c|c|c|c|c|c|c|c|}
\hline \multirow{2}{*}{ Lado } & \multirow{2}{*}{ Medida } & \multirow{2}{*}{\multicolumn{2}{|c|}{ Categorías }} & \multicolumn{2}{|c|}{ Esclerosis subcondral } & \multirow[t]{2}{*}{ Total } & \multirow{2}{*}{$P$} \\
\hline & & & & Ausente & Presente & & \\
\hline \multirow{6}{*}{ Derecho } & \multirow{6}{*}{ Ángulo ETR } & \multirow{2}{*}{ Alto $\left(60^{\circ}\right.$ a $\left.90^{\circ}\right)$} & n. ${ }^{\circ}$ & 0 & 0 & 0 & \multirow{6}{*}{0,860} \\
\hline & & & $\%$ & $0,00 \%$ & $0,00 \%$ & $0,00 \%$ & \\
\hline & & \multirow{2}{*}{ Moderado $\left(30^{\circ}\right.$ a $\left.60^{\circ}\right)$} & n. ${ }^{\circ}$ & 19 & 7 & 26 & \\
\hline & & & $\%$ & $73,10 \%$ & $26,90 \%$ & $100,00 \%$ & \\
\hline & & \multirow{2}{*}{ Bajo $\left(0^{\circ}\right.$ a $\left.30^{\circ}\right)$} & n. ${ }^{\circ}$ & 17 & 7 & 24 & \\
\hline & & & $\%$ & $70,80 \%$ & $29,10 \%$ & $100,00 \%$ & \\
\hline \multirow{6}{*}{ Izquierdo } & \multirow{6}{*}{ Ángulo ETR } & \multirow{2}{*}{ Alto $\left(60^{\circ}\right.$ a $\left.90^{\circ}\right)$} & n. ${ }^{\circ}$ & 0 & 0 & 0 & \multirow{6}{*}{0,723} \\
\hline & & & $\%$ & $0,00 \%$ & $0,00 \%$ & $0,00 \%$ & \\
\hline & & \multirow{2}{*}{ Moderado $\left(30^{\circ}\right.$ a $\left.60^{\circ}\right)$} & n. ${ }^{\circ}$ & 20 & 16 & 36 & \\
\hline & & & $\%$ & $55,60 \%$ & $44,40 \%$ & $100,00 \%$ & \\
\hline & & \multirow{2}{*}{ Bajo $\left(0^{\circ}\right.$ a $\left.30^{\circ}\right)$} & n. ${ }^{\circ}$ & 7 & 7 & 14 & \\
\hline & & & $\%$ & 50,00 & 50,00 & $100,00 \%$ & \\
\hline
\end{tabular}

Prueba de chi cuadrado

Tabla 14. Asociación entre el GRado del Ángulo ETR y LA PRESENCIA de QUiste subCondRal POR LADO

\begin{tabular}{|c|c|c|c|c|c|c|c|}
\hline Lado & Medida & \multicolumn{2}{|l|}{ Categorías } & \multicolumn{2}{|c|}{ Cuerpos libres } & Total & $P$ \\
\hline \multirow{6}{*}{ Derecho } & \multirow{6}{*}{ Ángulo ETR } & \multirow{2}{*}{ Alto $\left(60^{\circ}\right.$ a $\left.90^{\circ}\right)$} & n. ${ }^{\circ}$ & 0 & 0 & 0 & \multirow{6}{*}{0,267} \\
\hline & & & $\%$ & $0,00 \%$ & $0,00 \%$ & $0,00 \%$ & \\
\hline & & \multirow{2}{*}{ Moderado $\left(30^{\circ}\right.$ a $\left.60^{\circ}\right)$} & n. ${ }^{\circ}$ & 21 & 5 & 26 & \\
\hline & & & $\%$ & $80,80 \%$ & $19,20 \%$ & $100,00 \%$ & \\
\hline & & \multirow{2}{*}{ Bajo $\left(0^{\circ}\right.$ a $\left.30^{\circ}\right)$} & n. & 22 & 2 & 24 & \\
\hline & & & $\%$ & $91,70 \%$ & $8,30 \%$ & $100,00 \%$ & \\
\hline \multirow{6}{*}{ Izquierdo } & \multirow{6}{*}{ Ángulo EBT } & \multirow{2}{*}{ Alto $\left(60^{\circ}\right.$ a $\left.90^{\circ}\right)$} & n. ${ }^{\circ}$ & 0 & 0 & 0 & \multirow{6}{*}{0,514} \\
\hline & & & $\%$ & $0,00 \%$ & $0,00 \%$ & $0,00 \%$ & \\
\hline & & \multirow{2}{*}{ Moderado $\left(30^{\circ}\right.$ a $\left.60^{\circ}\right)$} & n. ${ }^{\circ}$ & 31 & 5 & 36 & \\
\hline & & & $\%$ & $86,10 \%$ & $13,90 \%$ & $100,00 \%$ & \\
\hline & & \multirow{2}{*}{ Bajo $\left(0^{\circ}\right.$ a $\left.30^{\circ}\right)$} & n. ${ }^{\circ}$ & 11 & 3 & 14 & \\
\hline & & & $\%$ & $78,60 \%$ & $21,40 \%$ & $100,00 \%$ & \\
\hline
\end{tabular}

Prueba de chi cuadrado 
TABLA 15. Asociación ENTRE EL GRAdo DEL ÁNGULO ETR Y LA PRESENCIA DE CUERPOS LIBRES POR LADO

\begin{tabular}{|c|c|c|c|c|c|c|c|}
\hline \multirow{2}{*}{ Lado } & \multirow{2}{*}{ Medida } & \multirow{2}{*}{\multicolumn{2}{|c|}{ Categorías }} & \multicolumn{2}{|c|}{ Cuerpos libres } & \multirow{2}{*}{ Total } & \multirow{2}{*}{$p$} \\
\hline & & & & Ausente & Presente & & \\
\hline \multirow{6}{*}{ Derecho } & \multirow{6}{*}{ Ángulo ETR } & \multirow{2}{*}{ Alto $\left(60^{\circ}\right.$ a $\left.90^{\circ}\right)$} & $n .^{\circ}$ & 0 & 0 & & \multirow{6}{*}{ nc } \\
\hline & & & $\%$ & $100,00 \%$ & $0,00 \%$ & $100,00 \%$ & \\
\hline & & \multirow{2}{*}{ Moderado $\left(30^{\circ}\right.$ a $\left.60^{\circ}\right)$} & n. ${ }^{\circ}$ & 26 & 0 & & \\
\hline & & & $\%$ & $100,00 \%$ & $0,00 \%$ & $100,00 \%$ & \\
\hline & & \multirow{2}{*}{ Bajo $\left(0^{\circ}\right.$ a $\left.30^{\circ}\right)$} & n. & 24 & 0 & & \\
\hline & & & $\%$ & $100,00 \%$ & $0,00 \%$ & $100,00 \%$ & \\
\hline \multirow{6}{*}{ Izquierdo } & \multirow{6}{*}{ Ángulo EBT } & \multirow{2}{*}{ Alto $\left(60^{\circ}\right.$ a $\left.90^{\circ}\right)$} & n. & 0 & 0 & 0 & \multirow{6}{*}{ nc } \\
\hline & & & $\%$ & $0,00 \%$ & $0,00 \%$ & $0,00 \%$ & \\
\hline & & \multirow{2}{*}{ Moderado $\left(30^{\circ}\right.$ a $\left.60^{\circ}\right)$} & n. ${ }^{\circ}$ & 36 & 0 & 36 & \\
\hline & & & $\%$ & $100,00 \%$ & $0,00 \%$ & $100,00 \%$ & \\
\hline & & \multirow{2}{*}{ Bajo $\left(0^{\circ}\right.$ a $\left.30^{\circ}\right)$} & n. ${ }^{\circ}$ & 14 & 0 & 14 & \\
\hline & & & $\%$ & $100,00 \%$ & $0,00 \%$ & $100,00 \%$ & \\
\hline
\end{tabular}

$\mathrm{nc}=$ no calculable

\section{DISCUSIÓN}

Conforme ha evolucionado la tecnología para en el diagnóstico por imágenes, la atención de los pacientes ha sido ha sido más oportuna y el diagnóstico ha sido más preciso; por lo tanto, la detección temprana de los defectos óseos adquiridos de la articulación temporomandibular a través de tomografía computarizada de haz cónico ha tomado mayor importancia. Los profesionales han utilizado la tomografía computarizada de haz cónico para realizar el diagnóstico de muchas patologías bucodentales y así evaluar el complejo maxilomandibular entero. Este trabajo se efectuó con la finalidad comparar la inclinación de la eminencia articular mediante tomografía computarizada de haz cónico de pacientes clase II esquelética, con y sin osteoartrosis, que acudieron al CDI de Lima, Perú, en el periodo 2010-2014. Se realizaron comparaciones con los últimos estudios realizados a fechas anteriores, en relación con la presencia en pacientes clase II con signos de osteoartrosis según sexo, lado y edad.

En este trabajo de investigación se obtuvo una muestra de TCHC de pacientes de acuerdo al rango de edad a estudiar. La muestra fue obtenida del CDI de Lima, Perú. Esta investigación propone ayudar al odontólogo de pregrado y posgrado a realizar un completo examen maxilomandibular por imágenes al paciente efectuando un diagnóstico certero y así proponer el tratamiento oportuno. La utilidad de la TCHC en la detección de defectos óseos adquiridos es evidente. La mayor eficacia diagnóstica para representar las erosiones condilares se encontró con el campo de visión más pequeño. En resumen, la TCHC en general tiene una precisión aceptable para el diagnóstico de anomalías óseas de la ATM, con una sensibilidad bastante alta $\left(^{1}\right)$.

Los trastornos de la articulación temporomandibular son la principal fuente de dolor orofacial de origen no dental. Los signos y síntomas clínicos más comunes de las DTM son dolor en las articulaciones, dolor muscular, limitación de la apertura bucal, chasquido y crepitación. Las DTM se asocian con frecuencia a cambios óseos degenerativos que afectan a las estructuras óseas de la articulación temporomandibular como aplanamiento, erosión, osteofitos, esclerosis 
ósea subcondral, cuerpos óseos libres y pseudoquistes. El conocimiento de estos cambios óseos es fundamental para el diagnóstico correcto de las disfunciones asociadas con la enfermedad y para la adecuada planificación del tratamiento $\left({ }^{48-60}\right)$.

En nuestro estudio, de un total de 50 pacientes, 21 eran hombres y 29 eran mujeres, y el promedio de edad fue de 30 años $\pm 6,13$, sin diferencias significativas entre sexos. El ángulo ANB usado como criterio de inclusión en promedio para toda la muestra fue de $7,02^{\circ} \pm 1,80^{\circ}$, sin diferencias significativas entre géneros.

Asimismo, no se encontraron diferencias significativas entre lados para la prevalencia de cambios osteoatrósicos. La mayor prevalencia fue del aplanamiento condilar, con el $82 \%$; la de osteofitos fue del $20 \%$; la erosión fluctuó entre el 50 $\%$ y el $56 \%$; la de esclerosis subcondral varió entre el $46 \%$ y el $72 \%$; la de quiste subcondral varió entre el $14 \%$ y el $16 \%$, y no se encontró ningún caso en que se presentaran cuerpos libres en ambos lados.

Estos resultados son parecidos a los reportados por Dos Anjos et al. $\left({ }^{2}\right)$, que en una muestra representativa hallaron una prevalencia de cambios óseos del $71 \%$. Koyama $\left({ }^{2}\right)$, por su lado, en un estudio realizado en $\mathrm{TCHC}$ encontró que el $63,7 \%$ de las articulaciones de los pacientes con DTM presenta cambios osteoatrósicos. Por otra parte, Capurso $\left({ }^{2}\right)$, en un estudio de frecuencias, reportó 14 cambios óseos degenerativos en el $19,6 \%$ de los pacientes evaluados.

Hintze $\left({ }^{6}\right)$, en un estudio de frecuencias que comparó el uso de la tomografía computarizada (TC) y la de haz cónico (TCHC), informó que ambas fueron eficaces para la identificación de defectos de aplanamiento y osteofitos, y que el aplanamiento condilar estaba presente en el $40 \%$ de la 159 de las articulaciones examinadas en promedio. Además, solo del $23 \%$ al $40 \%$ de sus hallazgos se identificaron correctamente mediante la evaluación de una combinación de imágenes frontal y sagital.

Para la asociación entre el grado del ángulo de la eminencia articular EBT y la presencia de cambios osteoatrósicos (aplanamiento, erosión, quistes subcondrales, osteofitos, esclerosis y cuerpos libres), en cada lado no se encontró asociación significativa entre alguna de estas categorías.

Para la asociación entre el grado del ángulo ETR y la presencia de cambios osteoartrósicos (aplanamiento, erosión, quistes subcondrales, osteofitos, esclerosis y cuerpos libres), en cada lado, tampoco se encontró asociación significativa entre alguna de estas categorías.

Zain-Alabdeen y Alsadhan ( $\left.{ }^{6}\right)$ reportaron que los valores medios de inclinación y altura de la eminencia articular fueron mayores en los varones que en las mujeres $(<0,05)$. En este mismo estudio, ellos también midieron el grosor del techo de la fosa glenoidea en relación con la morfología del cóndilo sagital y encontraron diferencias significativas. También, entre el grupo de pacientes con osteoartritis, el valor medio del espesor del techo de la fosa glenoidea para el grupo "osteoartritis-osteofito" fue el más alto $(1,59 \mathrm{~mm})$, mientras que los valores más bajos del techo de la fosa glenoidea se observaron en el grupo "osteoartritisaplanamiento".

Conforme al grado del ángulo ETR y la presencia de aplanamiento, se observó una asociación significativa en el lado izquierdo, con la categoría de ángulo bajo asociada a la presencia de aplanamiento en el $100 \%$ de los casos. La categoría de ángulo moderado presentó los cóndilos con aplanamiento en el $75 \%$ de 
los casos y con ausencia de este en el $25 \%$. Sin embargo, para darle un valor clínico a este resultado, el hallazgo debió notarse en ambos cóndilos, lo que no fue observado.

La asociación entre el ángulo ETR agrupado y la presencia de erosión mostró una asociación significativa en el lado derecho. Los pacientes con ángulo bajo presentaron erosión en el $83 \%$ de los casos, mientras que, en el ángulo moderado, alcanzaron el $30.80 \%$. Igualmente, este resultado no se observó en el lado izquierdo, situación que debe ser analizada desde el punto de vista clínico.

Se utilizan varios métodos de imagenología para evaluar los cambios óseos degenerativos que afectan la ATM $\left({ }^{60-65}\right)$. Es indispensable obtener imágenes claras y precisas de la región articular, aunque en algunas ocasiones esto podría dificultarse debido a que los individuos tienen diferentes angulaciones del cóndilo, algunos pacientes podrían tener limitación de la apertura bucal, o por la presencia de artefactos y movimientos mandibulares durante el examen imagenológico.

\section{CONCLUSIONES}

1. La prevalencia de osteofitos fue del $20 \%$; la de aplanamiento condilar, el $82 \%$; la erosión fluctuó entre el $50 \%$ y el $56 \%$; la de esclerosis subcondral varió entre el $46 \%$ y el $72 \%$; la de quiste subcondral varió entre el $14 \%$ y el $16 \%$, y no hubo ningún caso que presentara cuerpos libres en ambos lados.

2. Respecto de la inclinación de la eminencia articular, medida a través del ángulo EBT categorizado en tres grupos, no se encontró asociación significativa entre alguna de las categorías de cambios osteoatrósicos (aplanamiento, erosión, quistes subcondrales, osteofitos, esclerosis).

3. Con relación a la inclinación de la eminencia articular, medida a través del ángulo ETR categorizado en tres grupos, se encontró que la presencia de aplanamiento mostró una asociación significativa en el lado izquierdo, y para la presencia de erosión se encontró asociación significativa en el lado derecho.

4. En ninguna categoría de los ángulos EBT y ETR se presentaron cuerpos libres en ambos cóndilos.

Contribución del autor: Víctor Pardo Juárez ha participado en la concepción del artículo, la recolección de información, su redacción y la aprobación de la versión final.

Fuente de financiamiento: Autofinanciado.

Conflicto de interés: El autor declara no tener conflicto de interés de ningún tipo. 


\section{REFERENCIAS BIBLIOGRÁFICAS}

1. Larheim TA, Abrahamsson AK, Kristensen M, Arvidsson LZ. Temporomandibular joint diagnostics using CBCT. Dentomaxillofac Radiol. 2015; 44: 20140235.

2. Dos Anjos Pontual ML, Freire JSL, Barbosa JMN, et al. Evaluation of bone changes in the temporomandibular joint using cone beam CT. Dentomaxillofac Radiol. 2012; 41: 24-9.

3. Dilhan E, Mehmet I, Erdogan F, Semanur D, Nilüfer E. Articular eminence inclination, height, and condyle morphology on cone beam computed tomography. The Scientific World Journal. 2014; 761714.

4. Roque-Torres G, Meneses-López A, Bóscolo F. La tomografía computarizada cone beam en la ortodoncia, ortopedia facial y funcional. Rev Estomatol Herediana. 2015; 25 (1): 60-77.

5. Marinescu-Gava M. What the general dental practitioner should know about cone beam computed tomograph technology. OHDMBSC. 2009; VIII (4): 14-21.

6. Zain-Alabdeen EH, Alsadhan RI. A comparative study of accuracy of detection of surface osseous changes in the temporomandibular joint using multidetector CT and cone beam CT. Dentomaxillofac Radiol. 2012; 41: 185-91.

7. Liang X, Jacobs R, Hassan B, Li L, Pauwels R, Corpas L, et al. Acomparative evaluation of Cone Beam Computed Tomography (CBCT) and Multi-Slice CT (MSCT). Part I. On subjective image quality. Eur J Radiol. 2009; doi: 10.1016/j. ejrad.2009.03.042.

8. Jeffrey P. Okeson. Tratamiento de oclusión y afecciones temporomandibulares. 5a ed. Madrid: Mosby; 2003.

9. Alexiou KE, Stamatakis HC, Tsiklakis K. Evaluation of the severity of temporomandibular joint osteoarthritic changes related to age using cone beam computed tomography. Dentomaxillofac Radiol. 2009; 19225084.

10. Whaites E. Radiología odontológica. $2^{\underline{a}}$ ed. Buenos Aires: Médica Panamericana; 2010.

11. Krishnamoorthy B, Mamatha NS, Vinod Kumar AR. TMJ imaging by CBCT: Current scenario. Annals of Maxillofacial Surgery. 2013; 3 (1): 80-3.

12. Marques AP, Perrella A, Arita ES, Pareira MF, Cavalcanti Mde G. Assessment of simulated mandibular condyle bone lesions by cone beam computed tomography. Braz Oral Res. 2010; 24 (4): 467-74.

13. Perrella A, Borsatti MA, Peixoto Tortamano I, et al. Validation of computed tomography protocols for simulated mandibular lesions. A comparison study. Braz Oral Res. 2007; 21 (2): 165-9.

14. Boeddinghaus R, Whyte A. Computed tomography of the temporomandibular joint. Journal of Medical Imaging and Radiation Oncology. 2013; 57: 448-54. 
15. Barghan S, Tetradis S, Mallya SM. Application of cone beam computed tomography for assessment of the temporomandibular joints. Australian Dental Journal. 2012; 57 (1): 109-18.

16. Coskuner G, Ciger S. Three-dimensional assessment of the temporomandibular joint and mandibular dimensions after early correction of the maxillary arch form in patients with Class II division 1 or division 2 malocclusion. Korean J Orthod. 2015; 45 (3): 121-9.

17. Al-Saleh MAQ, Jaremko JL, Alsufyani N, Jibri Z, Lai H, Major PW. Assessing the reliability of MRI-CBCT image registration to visualize temporomandibular joints. Dentomaxillofac Radiol. 2015; 44: 20140244.

18. Nah KS. Condylar bony changes in patients with temporomandibular disorders: a CBCT study. Imaging Sci Dent. 2012; 42: 249-53.

19. Suomalainen A, Pakbaznejad Esmaeili E, Robinson S. Dentomaxillofacial imaging with panoramic views and cone beam CT. Insights Imaging. 2015; 6: 1-16.

20. Ozkan A, Altug HA, Sencimen M, Senel B. Evaluation of articular eminence morphology and inclination in TMJ internal derangement patients with MRI. Int. J. Morphol. 2012; 30 (2): 740-4.

21. Katsavrias EG, Changes in articular eminence inclination during the craniofacial growth period. Angle Orthod. 2002; 72: 258-64.

22. Kranjčić J, Vojvodić D, Žabarović D, Vodanović M, Komar D, Mehulić K. Differences in articular-eminence inclination between medieval and contemporary human populations. Arch Oral Biol. 2012; 57 (8): 1147-52.

23. Loubele M, Guerrero ME, Jacobs R, Suetens P, Steenberghe DV. A comparison of jaw dimensional and quality assessments of bone characteristics with ConeBeam CT, Spiral Tomography, and Multi-Slice Spiral CT. Int. J. Oral Maxillofac Implants. 2007; 22: 446-54.

24. Pauwels R, Beinsberger J, Collaert B, Theodorakou C, Rogers J, Walker A, et al. Effective dose range for dental cone beam computed tomography scanners. Eur $\mathrm{j}$ Radiol. 2012; 81: 267-71.

25. Suomalainen A, Kiljunen T, Käser Y, Peltola J, Kortesniemi M. Dosimetry and image quality of four dental cone beam computed tomography scanners compared with multislice computed tomography scanners. Dentomaxillofac Radiol. 2009; 38: 367-78.

26. De Vos W, Casselman J, Swennen G. Cone-beam computerized tomography (CBCT) imaging of the oral and maxillofacial region: a systematic review of the literature. Int J Oral and Maxillofac Surg. 2009; 38: 609-25.

27. Horner K, Islam M, Flygare L, Tsiklakis K, Whaites E. Basic principles for use of dental cone beam computed tomography: consensus guidelines of the European Academy of Dental and Maxillofacial. Dentomaxillofac Radiol. 2009; 38, 187-95.

28. Underhill TE, Chilvarquer I, Kimura K, Langlais RP, McDavid WD, Preece, JW, et al. Radiobiologic risk estimation from dental radiology. Part I. Absorbed doses to critical organs. Oral Surg Oral Med Oral Pathol. 1988; 66 (1): 111-20. 
29. Ngan DC, Kharbanda OP, Geenty JP, Darendeliler MA. Comparison of radiation levels from computed tomography and conventional dental radiographs. Aust Orthod J. 2003; 19 (2): 67-75.

30. Silva MA, Wolf U, Heinicke F, Bumann A, Visser H, Hirsch E. Cone-beam computed tomography for routine orthodontic treatment planning: a radiation dose evaluation. American J of Orthod and Dent Orthop. 2008; 18: 133: 640.

31. Choudhary AB, Motwani MB, Degwekar SS, Bhowate RR, Banode PJ, Yadav AO, et al. Utility of digital volume tomography in maxillofacial trauma. J Oral Maxillofac Surg. 2011; 69: e135-e140.

32. De Vos W, Casselman J, Swennen G. cone-beam computerized tomography (CBCT) imaging of the oral and maxillofacial region: a systematic review of the literature; International J Oral and Maxillof Surg. 2009; 38: 609-25.

33. Tsiklaklis K, Syriopouluos K, Stamatakis HC. Radiographic examination of TMJ using cone beam computed tomography. Dentomaxillofac Radiol. 2004; 33: 196-201.

34. Ludlow JB, Davies-Ludlow LE, Brooks SL. Dosimetry of two extraoral direct digital imaging devices: NewTom cone beam CT and Orthophos Plus DS panoramic unit. Dentomaxillof Radiol. 2003; 32: 229-34.

35. Pauwels $R$, et al. Effective dose range for dental cone beam computed tomography scanners. Eur j Radiol 2012; 81: 267-71.

36. Liu L, Li J, Ji H, Zhang N, Wang Y, Zheng G, et al. Cone-beam computed tomography evaluation of the maxillofacial features of patients with unilateral temporomandibular joint ankylosis undergoing condylar reconstruction with an autogenous coronoid process graft. PLoS One. 2017; 12 (3): e0173142.

37. Gorucu-Coskuner $\mathrm{H}$, Ciger S. Computed tomography assessment of temporomandibular joint position and dimensions in patients with class II division 1 and division 2 malocclusions. J Clin Exp Dent. 2017; 9 (3): e417-23.

38. Coskuner HG, Ciger S. Three-dimensional assessment of the temporomandibular joint and mandibular dimensions after early correction of the maxillary arch form in patients with Class II division 1 or division 2 malocclusion. Korean J Orthod. 2015; 45 (3): 121-9.

39. Sa SC, Melo SL, Melo DP, Freitas DQ, Campos PS. Relationship between articular eminence inclination and alterations of the mandibular condyle: a CBCT study. Braz. Oral Res. 2017; 31: e25.

40. Sümbüllü MA, Cağlayan F, Akgül HM, Yilmaz AB. Radiological examination of the articular eminence morphology using cone beam CT. Dentomaxillofac Radiol. 2012; 41, 234-40.

41. Horner K, Islam M, Flygare L, Tsiklakis K, Whaites E. Basic principles for use of dental cone beam computed tomography: consensus guidelines of the European Academy of Dental and Maxillofacial Radiology. Dentomaxillofac Radiol. 2009; 38 (4): 187-95. 
42. Caruso S, Storti E, Nota A, Ehsani S, Gatto R. Temporomandibular joint anatomy assessed by CBCT images. BioMed Research International. 2017; 2017: 2916953.

43. Tabrizi R, Shahidi S, Bahramnejad E, Arabion $\mathrm{H}$. Evaluation of condylar position after orthognathic surgery for treatment of class ii vertical maxillary excess and mandibular deficiency by using conebeam computed tomography. J Dent Shiraz Univ Med Sci. 2016; 17 (4): 318-25.

44. Saati S, Kaveh F, Yarmohammadi S. Comparison of cone beam computed tomography and multi slice computed tomography image quality of human dried mandible using 10 anatomical landmarks. J Clin Diagn Res. 2017; 11 (2): ZC13ZC16.

45. Al-Saleh MA, Punithakumar K, Lagravere M, Boulanger P, Jaremko JL, Wolfaardt $\mathrm{J}$, et al. Three-dimensional morphological changes of the temporomandibular joint and functional effects after mandibulotomy. Journal of Otolaryngology - Head and Neck Surgery. 2017; 46: 8.

46. Ma RH, Yin S, Li G. The detection accuracy of cone beam CT for osseous defects of the temporomandibular joint: a systematic review and meta-analysis. Sci. Rep. 2016; 6, 34714; doi: 10.1038/srep34714.

47. Al-Saleh MAQ, Jaremko JL, Alsufyani N, Jibri Z, Lai H, Major PW. Assessing the reliability of MRI-CBCT image registration to visualize temporomandibular joints. Dentomaxillofac Radiol. 2015; 44: 20140244.

48. Kyung-Soo Nah. Condylar bony changes in patients with temporomandibular disorders: a CBCT study. Imaging Sci Dent. 2012; 42: 249-53.

49. Naichuan Su, Yan Liu, Xianrui Yang, Jiefei Shen, Hang Wang. Correlation between oral health-related quality of life and clinical dysfunction index in patients with temporomandibular joint osteoarthritis. Journal of Oral Science. 2016; 58 (4): 483-90.

50. Levorová J, Machoń V, Guha A, Foltán R. Osteoarthrosis of temporomandibular joint related to the defects of posterior dentition: a retrospective study. Prague Medical Report. 2016; 117 (4): 176-84.

51. Massilla Mani F, Sivasubramanian SS. A study of temporomandibular joint osteoarthritis using computed tomographic imaging. Biomedical J. 2016; 39: 201-6.

52. Yang SW, Cho JY, Kim HM. Septic arthritis of the temporomandibular joint: a case report. J Korean Assoc Oral Maxillofac Surg. 2016; 42 (4): 227-30.

53. Gomes LR, et al. Diagnostic index of 3D osteoarthritic changes in TMJ condylar morphology. Proc SPIE Int Soc Opt Eng. 2015; 9414

54. Anderson WM, Marsh CM, Kessel NC, et al. Studying the prevalence and etiology of class ii subdivision malocclusion utilizing cone-beam computed tomography. $\mathrm{J}$ Orthod Endod. 2016, 2: 4.

55. Song Y, Zhang X, Yang Gao, Hou F, Yu Y. The condylar morphology in adult females of skeletal class II division 1 malocclusion with various vertical skeletal features: a study by cone beam computed tomography. Int J Clin Exp Med. 2016; 9 (5): 8304-11. 
56. Singh A, Sangur R, Lakshmana B, Mahajan T. A clinical study to determine the pattern of occlusal contacts in lateral positions and its validity in classifying guidance patterns. J Indian Prosthodont Soc. 2013; 13 (2): 101-7.

57. Gnanashanmugham K, Saravanan B, Sukumar MR, Faisal T. Gnathological splint therapy in temporomandibular joint disorder. J Pharm Bioallied Sci. 2015; 7 (Suppl 1): S314-S318.

58. Wolford LM. Can orthodontic relapse be blamed on the temporomandibular joint? J Orthod Sci. 2014; 3 (4): 95-105.

59. Ballanti F, Ranieri S, Baldini A, Pavoni Ch, Bollero P, Cozza P. Gnathological features in growing subjects. Annali di Stomatol. 2013; IV (3-4): 230-8.

60. Funato M, Ono Y, Baba K, Kudo Y. Evaluation of the non-functional tooth contact in patients with temporomandibular disorders by using newly developed electronic system. J of Oral Rehab. 2014; 41: 170-6.

61. Kaidonis JA, Ranjitkar S, Lekkas D, Brook AH, Townsend GC. Functional dental occlusion: an anthropological perspective and implications for practice. Austral Dent J. 2014; 59: (1 Suppl): 162-73.

62. Felício CM, Melchior Mde O, Silva MA, Celeghini RM. Masticatory performance in adults related to temporomandibular disorder and dental occlusion. Pró-Fono. 2007; 19 (2): 151-8.

63. Davies SJ, Gray RM, Sandler PJ, O'Brien KD. Orthodontics and occlusion. British Dental J. 2001; 191: 539-49.

64. Čimić S, Žaja M, Šimunković SK, Kopić M, Čatić A. Influence of occlusal interference on the mandibular condylar position. Acta Stomatol Croat. 2016; 50 (2): 116-21.

65. Skirbutis G, Surna A, Barauskas R, Surna R, Gleiznys A. A revised approach of human mastication function rehabilitation through monotypical mastication analysis. Stomatologija, Baltic Dental and Maxillofac J. 2015; 17: 13-20. 